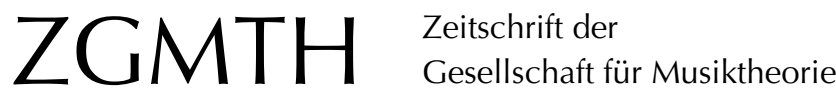

Menke, Johannes (2006): Kontrapunkt im 17. Jahrhundert - ein Lehrgang. ZGMTH 3/3, 341-353. https://doi.org/10.31751/239

(C) 2006 Johannes Menke

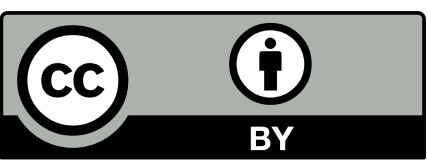

Dieser Text erscheint im Open Access und ist lizenziert unter einer Creative Commons Namensnennung 4.0 International Lizenz.

This is an open access article licensed under a

Creative Commons Attribution 4.0 International License.

veröffentlicht / first published: 01/07/2006 zuletzt geändert / last updated: 17/12/2008 


\title{
Kontrapunkt im 17. Jahrhundert - ein Lehrgang
}

\author{
Johannes Menke
}

Präsentiert wird ein einsemestriger Lehrgang über kontrapunktische Satztechnik im 17. Jahrhundert. Entscheidend für diesen Zeitraum ist nicht die Komplexität des Kontrapunktischen an sich, sondern die Integration neuer Techniken wie Figuren, Figuration, Sequenz, Chromatik und Variation in die aus der Renaissance tradierte Satztechnik. Dies kann anhand des zweistimmigen Choralvorspiels, der dreistimmigen Partita oder der vierstimmigen Toccata geübt und erlernt werden.

Im gegenwärtigen Kontrapunktunterricht dominiert die Beschäftigung mit der Musik des 16. und 18. Jahrhunderts. ${ }^{1}$ Die Musik des 17. Jahrhunderts dagegen findet nicht die Beachtung, die sie verdient. Im 17. Jahrhundert fanden jedoch höchst folgenreiche satztechnische Umwälzungen statt: Die Musiksprache der barocken Epoche ist weitgehend ein Produkt des 17. Jahrhunderts und ohne dessen Kenntnis schlechterdings nicht verständlich. Ferner ist zu bedenken, dass die aus dem 17. Jahrhundert erwachsene barocke Harmonik (Generalbass) und Satztechnik (Kontrapunkt) die maßgeblichen Disziplinen in der Komponisten-Ausbildung auch noch im 19. und auch im 20. Jahrhundert waren. ${ }^{2}$ Schließlich vermag die Kenntnis der Kompositionstechnik des 17. Jahrhunderts die Lücke zwischen klassischer Vokalpolyphonie und barocker instrumentaler Polyphonie zu schließen und damit das Verständnis beider zu erhellen.

Ausgehend von diesen Überlegungen habe ich für das Sommersemester 2005 eine satztechnische Übung »Kontrapunkt 17. Jahrhundert« entwickelt, die seitdem regelmäßig stattfindet und die ich hiermit zur Diskussion stellen möchte. Um Missverständnissen vorzubeugen, möchte ich darauf hinweisen, dass es im Folgenden nicht um Forschung über barocke Satztechnik geht, sondern um didaktische Vorüberlegungen und methodische Entwürfe.

1 Auf dem IV. Kongress der GMTH 2004 in Köln hielt ich einen Vortrag, in dem ich dafür plädierte, die Musik des 17. Jahrhunderts in den Kontrapunktunterricht zu integrieren (Menke 2004). Es ging mir dabei nicht darum, Kanonbildung als solche in Frage zu stellen, sondern auf ein Defizit hinzuweisen.

2 Man denke nur an den gut dokumentierten Kompositionsunterricht Beethovens (vgl. Nottebohm 1873). 


\section{Prämissen}

Stilkopien können sich auf alle möglichen Personal- oder Epochenstile beziehen. Niemand kann sich aber in allen Stilen gleichermaßen gut auskennen. Es ist also unumgänglich, eine Auswahl zu treffen, ja mehr, Prioritäten zu setzen. Die Leitfrage dabei sollte meines Erachtens immer die nach dem spezifischen `Beitrag des Stils sein: Welche entscheidenden Neuerungen, die auch für nachfolgende Generationen wichtig wurden, hat ein Stil etabliert? Jede Erfahrung, die man mit Stilkopien macht, ist eine Erfahrung mit der Geschichtlichkeit des musikalischen Materials ${ }^{3}$ und den ihm jeweils innewohnenden Potentialen und Problemstellungen.

Was also ist der Beitrag des 17. Jahrhunderts? Diese pauschale Frage können wir hier natürlich nicht definitiv beantworten, aber doch immerhin so weit, dass entschieden werden kann, mit welchen Inhalten man einen satztechnischen Lehrgang füllt.

Für viele ist das 17. Jahrhundert nicht gerade eine Blütezeit kontrapunktischen Komponierens. ${ }^{4}$ Zumindest vermittelt die Schwerpunktbildung im satztechnischen Fächerkanon das Bild, als seien die klassische Vokalpolyphonie und die spätbarocke instrumentale Polyphonie zwei unangefochtene Höhepunkte, die sich recht unvermittelt gegenüberstehen: Der lineare Kontrapunkt auf der einen und der harmonische auf der anderen Seite. Der sstile antico< aber ist nichts Statisches, sondern wurde im Laufe des 17. und 18. Jahrhunderts durch eine Vielzahl von Techniken bereichert. Am Ende seiner Entwicklung, also etwa bei Fux oder Albrechtsberger, ging es nicht um Stilkopien der Palestrinazeit, sondern um kontrapunktische Gerüstsätze, die ihre Wurzeln gleichwohl in der Palestrinazeit und der Contrapunctus-Lehre des 15. und 16. Jahrhunderts haben. Darüber hinaus gibt es im 17. Jahrhundert eine Tendenz, den kontrapunktischen Satz aufzulockern, oft sogar in Figuration aufzulösen, jedenfalls immer zu differenzieren und anzureichern. Das 17. Jahrhundert erfindet keine neuen kontrapunktischen Techniken, aber - und darauf kommt es an - es tradiert die alten und integriert in sie ganz neue, bis heute wirksame Techniken: Figuren, Figuration, Sequenz, Chromatik und Variation. Dies sei im Folgenden kurz erläutert.

- Figur. Ungeachtet der Fragwürdigkeit des Begriffs der $>$ Figurenlehre $\iota^{5}$ sind Figuren in satztechnischer Hinsicht vor allem insofern bedeutend, als sie bestimmte expressiv aufgeladene und zugleich standardisierte Formen von Dissonanzbehandlung bezeichnen, in denen die Musik des 17. Jahrhunderts vom klassischen System abweicht. Mag man auch die `deutsche`Gründlichkeit eines Christoph Bernhard beargwöhnen - sein Verdienst ist es, einen Versuch unternommen zu haben, dies ganz klar zu benennen.

3 So problematisch er inzwischen vielen zeitgenössischen Komponisten erscheinen mag, erweist der von Th. W. Adorno geprägte Begriff des »musikalischen Materials« seine Plausibilität m. E. doch in Hinblick auf historische Stile: So lässt sich relativ genau feststellen, welche Gattungen, Satztechniken, Topoi, Akkordformen, Dissonanzbehandlungen etc. spezifisch für den `Stand des Materials` in einer bestimmten Zeit und in einem bestimmten Raum waren.

4 Dass sich kontrapunktisches Komponieren nur in Sätzen, die dem Prinzip der Imitation folgen (Kanon, Fuge etc.), manifestiere, ist eine verengte Ansicht, die zuweilen zu einer solchen Einschätzung führen kann.

5 Siehe dazu die Beiträge von Janina Klassen und Bettina Varwig in dieser Ausgabe. 
- Figuration. Seit dem 17. Jahrhundert kommt der kompositionstechnischen Unterscheidung von Gerüstsatz und dessen Diminution oder Figuration eine noch größere Bedeutung als vorher zu. Gerüstsatz kann ein klassischer kontrapunktischer Satz sein oder auch ein rein homophoner Satz. Mit der Figuration hält das virtuose und ornamentale Element Einzug in die Kompositionstechnik ${ }^{6}$, ihre prototypische Gattung ist die Toccata. Nach und nach werden alle Gattungen und Satztechniken mit figurativen und ornamentalen Elementen durchsetzt.

- Sequenz. Die Forderung der Renaissance-Ästhetik nach varietas impliziert eine bewusste Vermeidung sequenzartiger Strukturen. Komponisten der Josquin-Generation verwendeten Sequenzen mit rhetorischer Intention als bewusste Abweichung von der varietas. Mit dem 17. Jahrhundert wird die Sequenz erstmals integraler Bestandteil der Musiksprache. Unterschiedliche bassbezogene Sequenztypen werden entwikkelt und dominieren die harmonische Logik der Epoche. ${ }^{7}$ Schon bei Jan Pieterszoon Sweelinck kann man fast alle diatonischen Sequenztypen nachweisen, die bis heute verwendet werden. Die im 19. Jahrhundert einsetzende Verachtung der Sequenztechnik (`Schusterflecken`) hat deren Bedeutung leider etwas vergessen lassen. ${ }^{8}$

- Chromatik. Während im 16. Jahrhundert mit Chromatik nur experimentiert wurde (man denke an Lassos berühmte Prophetiae Sibyllarum, die in seinem œuvre bezeichnenderweise ein Einzelfall sind), war es den Komponisten des 17. Jahrhunderts ein Anliegen, eine in sich schlüssige Form chromatischer Harmonik zu etablieren. Auch hier wurden Maßstäbe gesetzt, die weit über das Jahrhundert hinaus gültig gewesen sind. Das Prinzip, demzufolge Chromatik - ganz im griechischen Wortsinn - als Färbung eines diatonischen Satzes zu verstehen ist, gilt für Gesualdo und Frescobaldi, war noch für Simon Sechter, wenngleich unter veränderten Voraussetzungen, gültig und erstreckt sich selbst noch auf weite Teile der spätromantischen Harmonik.

- Variation. Die Variation, wie sie sich vornehmlich in der Gattung der Partita entfaltet, ist bereits eine Errungenschaft des späten 16. Jahrhunderts. Schon das berühmte Fitzwilliam Virginal Book stellt ein beeindruckendes Kompendium der Variationskunst dar. Kompositionstechnisch folgenreich ist in diesem Zusammenhang nicht nur das Verfahren der Variation, mit all seinen Querbeziehungen zur Figuration, sondern

6 Die Verachtung des Ornaments in der Ästhetik des 20. Jahrhunderts (vgl. z. B. Adolf Loos, Ornament und Verbrechen, 1908), die sich im ästhetischen Unbewussten mancherorts festgesetzt zu haben scheint, hat sicherlich dazu beigetragen, diesen Aspekt, der eine wahre Revolution gewesen sein muss, geringzuschätzen.

7 Viele Sequenztypen entstammen bereits dem 15. Jahrhundert, wie etwa aus dem berühmten Traktat De preceptis artis musicae von Guilielmus Monachus zu ersehen ist. Wenngleich viele Modelle tradiert wurden, scheint mir die konsequente Entfaltung der Sequenz doch eine Leistung des 17. Jahrhunderts zu sein. Bedingt durch die Dominanz des Generalbasses erfolgte die Systematisierung von Sequenzen nicht mehr ausgehend von Stimmpaaren, sondern vom Bass aus. Wichtige Quellen für diese folgenreiche Denkweise sind Bianciardi 1607 und Spiridionis 1670/71.

8 Die Verachtung der Sequenz rührt zu großen Teilen von Schönberg und seiner Schule her. In seinem Aufsatz Kriterien für die Bewertung von Musik (Schönberg 1946) stellt er der minderwertigen Sequenztechnik, die er unter anderem bei Wagner vorfindet, die höherstehende und fortschrittlichere Variationstechnik gegenüber. Dies hat aufgrund der großen Autorität Schönbergs, insbesondere nach 1945, zu bestimmten Ausblendungen in der Theorie und zur Geringschätzung bestimmter Komponisten (z. B. Bruckner) geführt. 
auch die Etablierung bassbezogener harmonischer Topoi wie Romanesca, Folia, Passamezzo usw.

Selbstverständlich beschränkt sich der Beitrag des 17. Jahrhunderts kompositionstechnisch nicht auf die genannten fünf Punkte. Ich denke aber doch, dass damit einige wesentliche Aspekte berührt werden.

\section{Ziele und Vorgehensweise}

Auf diesem Hintergrund nun lassen sich Inhalte und Ziele eines satztechnischen Lehrgangs näher bestimmen.

Vorausgesetzt, die genannten Techniken (Figuren, Figuration, Sequenz, Chromatik und Variation) machen den wesentlichen kompositionstechnischen Beitrag aus, kommt es darauf an, die Techniken analytisch aufzuspüren, sie zunächst isoliert zu üben, um sie dann in den formalen Zusammenhang eines vollständigen Stückes zu stellen. Die Methoden können vielfältiger Art sein:

- Höranalyse

- Analyse von Notentexten

- Übung nach bestimmten, aus der Analyse gewonnenen Vorgaben

- Übung mit Gerüstsätzen

- Übung mit Lückentexten

- Improvisation etc.

Abschluss des Lehrgangs ist ein Vortragsabend, in dem die entstandenen Arbeiten aufgeführt werden. Dieses künstlerische Ziel stachelt den Ehrgeiz an, es lässt die Studenten in die Rolle der Komponisten schlüpfen und unterstreicht die künstlerische Dimension des Faches Musiktheorie.

\section{Lehrgang}

Sind Inhalte und Methoden grob umrissen, so muss entschieden werden, an welche Gattungen man sich hält. Diese Entscheidung ist stark davon abhängig, an welche Zielgruppe sich eine satztechnische Übung richtet. In meinem Fall handelt es sich um Studenten der Studiengänge 'Künstlerische Ausbildung` und `Musiklehrer` mit unterschiedlichen Instrumentalhauptfächern ab dem 5. Semester, die Vorkenntnisse im Bereich der Harmonielehre besitzen (Generalbass und Choralsatz sowie analytische Erfahrungen), aber keine im Bereich des Kontrapunkts. Gewisse Grundkenntnisse in bezug auf den klassischen Kontrapunkt müssen also zunächst vermittelt werden, um dann nach und nach Techniken des 17. Jahrhunderts einzuführen und damit behutsam zu immer anspruchsvolleren Aufgabenstellungen zu gelangen. Neben technischen Fragen ist es unabdingbar, sich 
mit der Stilistik und damit der Ästhetik der Zeit vertraut zu machen - am besten durch Hörbeispiele und eigenes Musizieren.

Um dem zeitlichen Rahmen eines Semesters nicht zu sprengen, habe ich mich dafür entschieden, mich auf drei Gattungen zu beschränken. ${ }^{9}$ Anhand dieser Gattungen sollen kontrapunktisches Schreiben und die genannten Techniken unter kontinuierlicher Zunahme des Schwierigkeitsgrades erlernt werden.

\section{Einführung}

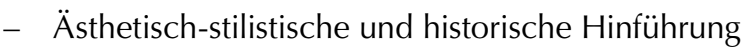

- Technische Grundlagen: Modi, Intervalle, Klauseln, Rhythmus, Syncopatio

II. Choralvorspiel, zweistimmig nach dem Vorbild von Jan Pieterszoon Sweelinck

- Vertiefung: Figuren als erweiterte Dissonanzbehandlung

III. Exkurs: Chromatik, nach dem Vorbild von Giacomo Carissimi

- Modelle, die zwei- und dreistimmig ausgeführt werden

IV. Partita, dreistimmig, nach verschiedenen Vorbildern (Claudio Monteverdi, Girolamo Frescobaldi)

- Improvisation über die Bergamasca

- Romanesca, unter besonderer Berücksichtigung des Außenstimmensatzes

- Vertiefung: Harmonik im 17. Jahrhundert, Gebrauch von Sextakkorden

- Ciaccona

V. Toccata, vierstimmig, nach dem Vorbild von Jan Pieterszoon Sweelinck

- Form, nach dem Muster antiker Rhetorik: Exordium, Narratio, Argumentatio, Conclusio $^{10}$ Frequenz der Harmoniewechsel und Dramaturgie

- Formale Funktionen von Harmonik

- Sequenzmodelle und deren Figuration

- Echo-Technik

- Vergleichender Blick: Girolamo Frescobaldi

9 Harold Owen schlägt in seinem umfassenden und anregenden Kontrapunktlehrbuch (Owen 1992) für das 17. Jahrhundert folgende Gattungen vor: Rezitativ, Variation, Trio-Satz, Vorgänger-Formen der Fuge.

10 Ueding 1995, $72 \mathrm{ff}$. 


\section{Kommentar}

Selbstverständlich kann ich den Lehrgang hier nicht vollständig vorstellen. Ich werde mich darauf beschränken, die einzelnen Punkte des Planes kurz zu kommentieren.

\section{Einführung}

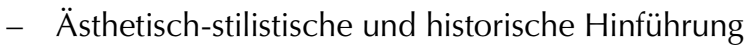

Da bei vielen Studenten erfahrungsgemäß wenige Vorkenntnisse in bezug auf die Musik svor Bach vorhanden sind, werden je ein Kyrie von Giovanni Pierluigi Palestrina und Giacomo Carissimi verglichen. Ästhetische Kriterien der Renaissance- und Barockmusik werden benannt und anhand weiterer Hörbeispiele vertieft.

- Technische Grundlagen: Modi, Intervalle, Klauseln, Rhythmus

Hier ist es nötig, den Stand vor 1600 aufzuzeigen, um dann auf einige tiefgreifende Änderungen hinzuweisen, die im Laufe des 17. Jahrhunderts stattgefunden haben so beispielsweise die Erweiterung und letztliche Ablösung der Modi durch Dur und Moll, die Beibehaltung der Intervallklassen (perfekt, imperfekt, dissonant), aber die Modifikationen bestimmter Techniken der Dissonanzauflösung (etwa die Auflösung der Quarte in die Sexte usw.) bzw. die Einführung verminderter und übermäßiger Intervalle und deren Behandlung, die Verzierung der Klauseln oder das Aufkommen des »Akzentstufentaktes» (Besseler) und die Einführung des Bindebogens.

\section{Choralvorspiel, zweistimmig nach dem Vorbild von Jan Pieterszoon Sweelinck}

Das zweistimmige Choralvorspiel im Stil von Sweelinck eignet sich ideal, um einen Einstieg in kontrapunktisches Schreiben zu finden ${ }^{11}$ : Eine Stimme ist vorgegeben, die andere Stimme ist nach bestimmten rhythmischen Mustern, die den Fux'schen Gattungen ähneln, zu gestalten, die Dissonanzbehandlung ist recht einheitlich und man lernt, intervallisch zu denken, was für die Studierenden vor allem dann eine enorm horizonterweiternde Erfahrung ist, wenn sie bislang nur Erfahrung mit der reduzierten Welt einer reinen `Harmonielehre` gemacht haben. Schon beim zweistimmigen Choralvorspiel können Akkordfiguration, erweiterte Dissonanztechnik (Figuren) und vor allem Sequenzbildung geübt werden.

- Vertiefung: Figuren als erweiterte Dissonanzbehandlung

Um bestimmte schnelle Passagen oder bestimmte Kadenzbildungen zu verstehen, ist die Kenntnis wichtiger Figuren nötig, so etwa der 'Superjectio oder der `Sincopa tutta cattivar.

11 Als Beispiele eignen sich gut (in dieser Reihenfolge):

- Es spricht der Unweisen Mund wohl

- Da pacem, Domine, in diebus nostris und

- Nun komm der Heiden Heiland 


\section{Exkurs: Chromatik und Figuren nach dem Vorbild von Giacomo Carissimi}

Für das richtige Verständnis und den adäquaten Einsatz von Chromatik sind zwei Erkenntnisse hilfreich: Erstens ist der chromatische Schritt vom halbtönigen zu unterscheiden: Durch Chromatik werden ganztönige Schritte 'gefärbt‘; chromatische Passagen sind immer reduzierbar auf diatonische Vorgänge und aus dieser `Schwarz-weiß-Fassung, heraus erklärbar. Zweitens ist die Behandlung von Chromatik meist standardisiert, sowohl hinsichtlich des Skalenausschnitts und der Bewegungsrichtung (prominentestes Beispiel ist der Lamentobass), als auch, was die Intervall-, oder, in der Mehrstimmigkeit, die Akkordstruktur betrifft.

Dieses Thema bietet Gelegenheit, einen Komponisten vorzustellen, dessen einstige Bedeutung leider in umgekehrt proportionalem Verhältnis zu seiner heutigen Bekanntheit steht: Giacomo Carissimi. Bei ihm lässt sich der wirkungsvolle Einsatz von Figuren, zu denen auch die Chromatik gehört (‘passus duriusculus`, wie es sein Schüler Christoph Bernhard genannt hat), beobachten.

Mit Hilfe eines Lückentextes nach Carissimi lassen sich Chromatik und Figuren bestens üben:
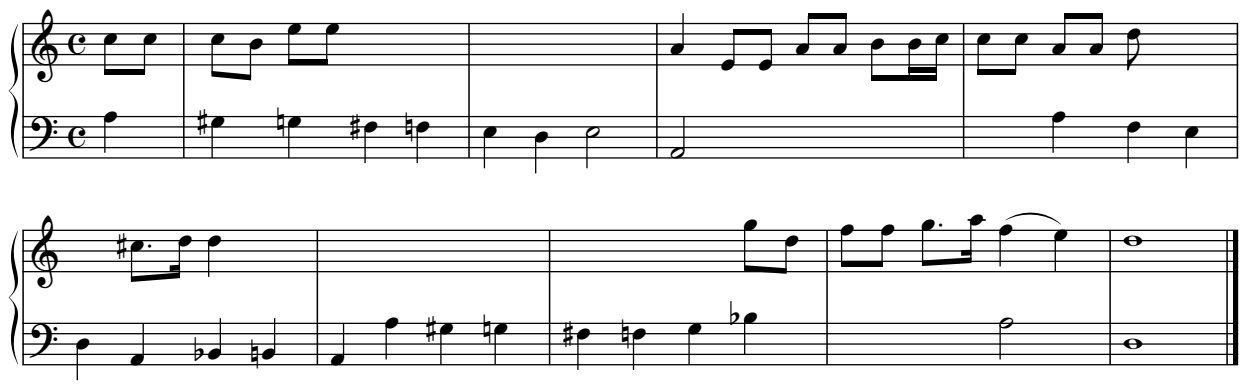

Beispiel 1: Lückentext nach einem Ausschnitt aus dem Oratorium Iudicium Salomonis von Giacomo Carissimi

Eine andere Übung kann darin bestehen, einen gegebenen einfachen Satz, der im wesentlichen den Intervallsatz-Regeln eines barockisierten stile antico folgt, mit Figuren auszuzieren. Die Figurentypologie von Bernhard erweist sich als brauchbarer Katalog für ein solches Vorhaben (siehe Beispiel 2).

Durch die `Anreicherung eines gegebenen zweistimmigen chromatischen Außenstimmensatzes mit einer Mittelstimme gelangt man recht gut zur Dreistimmigkeit. Als Modell für die Klangfortschreitung bietet sich der Chor »Et ululantes filii Ammon« aus dem Oratorium Jephte von Carissimi an. 


\section{Partita, dreistimmig, nach verschiedenen Vorbildern (Monteverdi, Frescobaldi)}

- Romanesca, unter besonderer Berücksichtigung des Außenstimmensatzes

Schon bei der Chromatik zeigt sich die Tendenz zur Standardisierung der Satztechnik in Form von bis ins Detail hinein modellhaft vorgefertigten Klangfortschreitungen. Die häufige Verwendung von Satzmodellen darf nicht als Depravation missverstanden werden; vielmehr ist sie ein Symptom für die Idiomatisierung von Musik im Barockzeitalter. Komponieren heißt nicht, wie es die ästhetische Ideologie des 19. Jahrhunderts forderte, etwas Neues aus dem Nichts heraus zu erschaffen, sondern die übernommene Sprache originell, expressiv, aber vor allem verständlich zu sprechen. ${ }^{12}$ Anhand der Romanesca lässt sich exemplarisch studieren, dass ein Bassmodell nicht nur eine bestimmte Klangfortschreitung im Sinne von Akkordfolge generiert, sondern einen ganz bestimmten Verlauf der Melodiestimme provoziert. ${ }^{13}$ Erstaunlich ist, welch unterschiedliche Ausprägungen ein und derselbe Gerüstsatz erfahren kann.
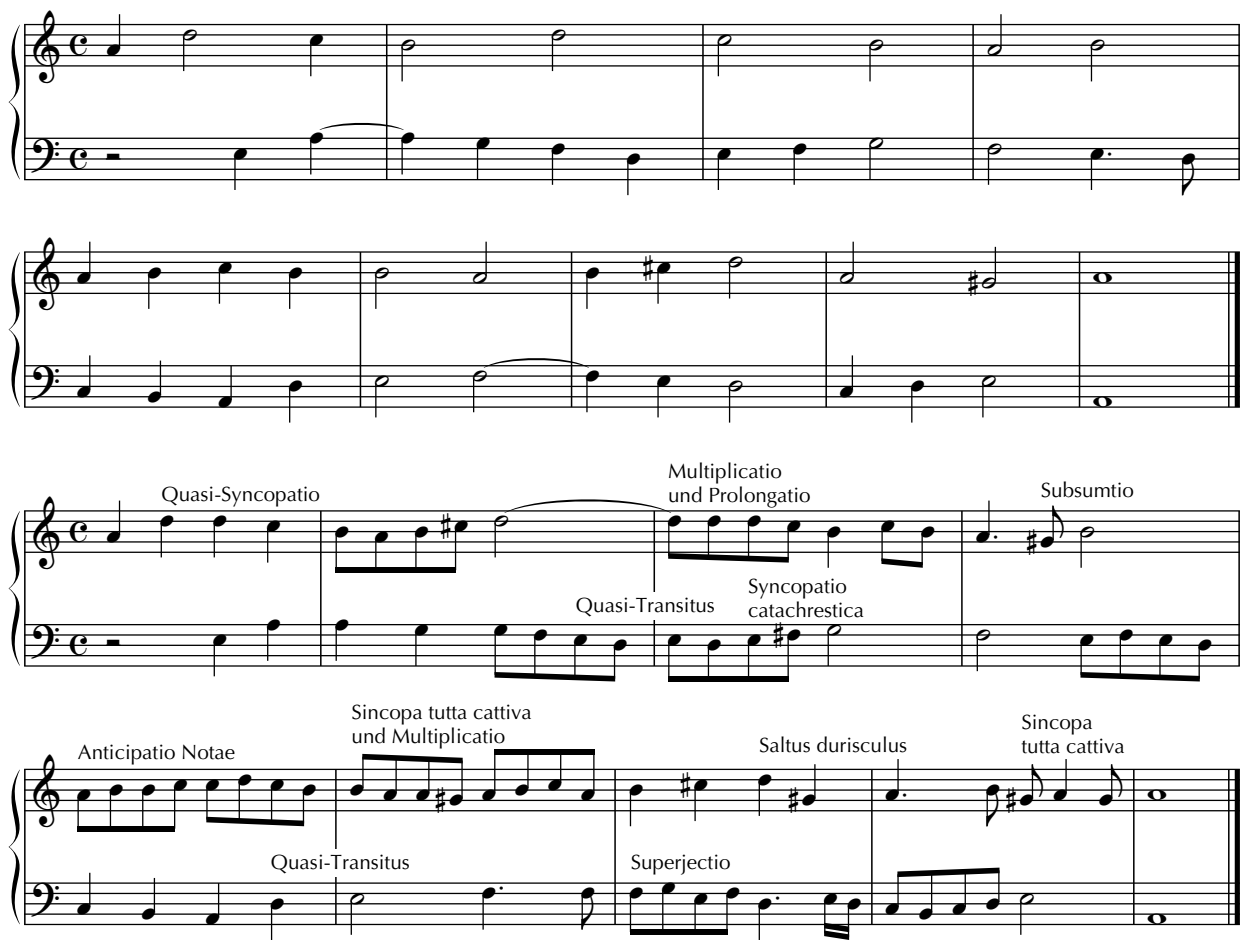

Beispiel 2: Einfacher Gerüstsatz im stile antico und Auszierung dieses Satzes mit Figuren

12 Hierzu auch der Text von Janina Klassen in dieser Ausgabe.

13 Dass hier ein Zugriff nach Schenker'schem Muster (Quintzug in der Oberstimme $d$-g) einsetzen könnte, ist offensichtlich, kann hier aber nicht weiter ausgeführt werden. 

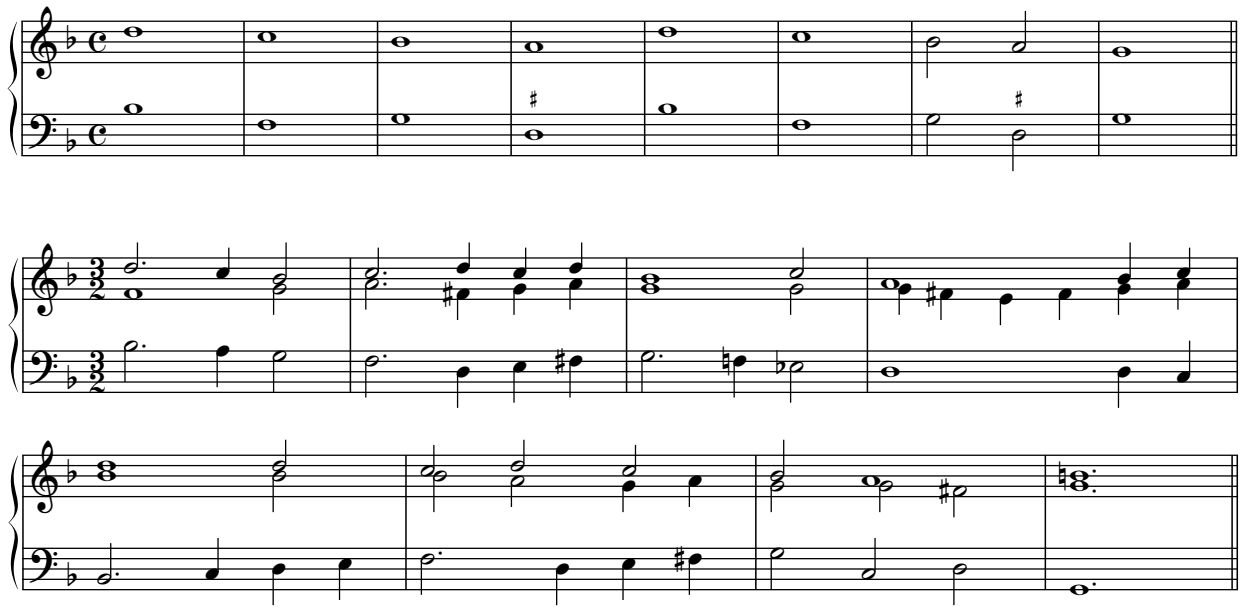

Beispiel 3: Gerüstsatz der Romanesca und einfache Ausarbeitung

Ein Vergleich des Madrigals Ohimè dov'è il mio ben von Monteverdi mit den Partite 14 sopra I'Aria della Romanesca von Frescobaldi zeigt, wie unterschiedlich eine `Ausformulierung` des Romanesca-Modells aussehen kann; Bassschritte können chromatisiert und/oder ausgefüllt, Harmonien mit Dissonanzbildungen angereichert und Melodien schließlich trotz der klaren Gerüsttöne ganz unterschiedlich variiert werden.

Die Klangfortschreitung der Romanesca mit ihrem Terz-Quint-Außenstimmensatz entspricht einem seit dem späten Mittelalter gültigen Grundmuster, welches auch dem omnipräsenten Folia-Modell zugrunde liegt. Dieses Beziehungsgeflecht fasziniert die Studenten immer wieder; vermutlich auch deshalb, weil sie - je nachdem, wie sie vorgeprägt sind - zu ahnen beginnen, dass es noch andere `Urformeln als Tonika, Dominante und Subdominante.

- Vertiefung: Harmonik im 17. Jahrhundert, Gebrauch von Sextakkorden

Da der Bass in den gewählten Gattungen meist nach bestimmten Mustern verläuft (Passacaglia oder Sequenz) und die vorkommenden Klänge - von den anderweitig regulierten Dissonanzbildungen abgesehen - nur Dreiklänge und Sextakkorde sind, ist nur die Frage zu beantworten, wo Sextakkorde stehen sollen bzw. müssen. Schon im 17. Jahrhundert gilt im wesentlichen Gasparinis klassisches Diktum, demzufolge die kleine Sexte über dem Basston anstelle der Quinte dann gesetzt wird, wenn der Bass »einen Halbton, sei es diatonisch oder durch Versetzungszeichen bedingt ansteigt«. ${ }^{14}$ Dies ergibt eine Oktavregel mit Sextakkorden auf der 3. und 7. BassStufe in Dur. Die Oktavregel in Moll lässt sich entweder analog dazu bilden oder

14 »Quando ascende la nota il grado di un semitono, o naturale, o accidentale [...], alla prima li si dà la Sesta minore.« (Gasparini 1708, 18) 
aber auch mit einem Sextakkord über der 2. und einem Dreiklang über der 3. Stufe. Neben dieser >Mindestausstattung` mit Sextakkorden gehören auch die in der späteren sregola dell'ottava kodifizierten Sextakkorde über der 2. und 6. Skalenstufe (bei stufenweiser Fortschreitung des Basses) im 17. Jahrhundert zum Standardrepertoire. Selbstverständlich sind solche Abstraktionen nur Annäherungswerte, sie garantieren aber einen einigermaßen sicheren Umgang mit Harmonik, ohne dass Harmonielehre-Systeme des 19. Jahrhunderts bemüht werden müssen. Daneben ist zu beachten, dass Sextakkorde im Rahmen von Consecutiven und Sequenzen anderweitig eingesetzt werden. Schon im 17. Jahrhundert kann davon ausgegangen werden, dass es unterschiedliche >Modi von Harmonik gibt, die sich, wie W. E. Caplin in bezug auf das klassisch-romantische Repertoire formuliert hat, auf die Funktionen Prolongation, Sequenz und Kadenz beziehen. ${ }^{15}$

- Ciaccona

Während im Grundmodell der Romanesca gar keine Sextakkorde vorgesehen sind, liegt der Reiz des Ciaccona-Basses gerade darin, dass der Sextakkord über der 3. Stufe in Dur angesprungen wird. Ein reizvolles Beispiel für die Ciaccona ist Monteverdis abwechslungsreiches und kunstvolles Madrigal Zefiro torna. Man kann gut erkennen, dass es hier im Gegensatz zur Romanesca keine modellhaft festgelegte Oberstimme gibt, sondern unterschiedliche Verläufe möglich sind, die zunächst erprobt und dann figuriert werden können.

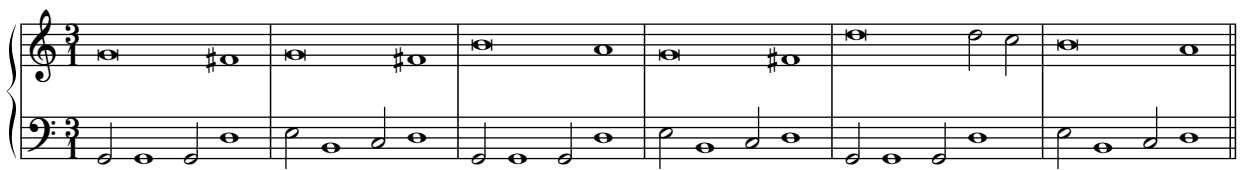

Beispiel 4: Bass der Ciaccona nach Monteverdis Zefiro torna mit modellhaften Oberstimmenverläufen in Oktav-, Terz- und Quintlage

Die Kontinuität barocker Komponierpraxis zeigt sich darin, dass Bach und Händel eine spätere Variante des Ciaccona-Basses benutzt haben, wobei die fallende Quarte am Anfang stufenweise ausgefüllt wird:

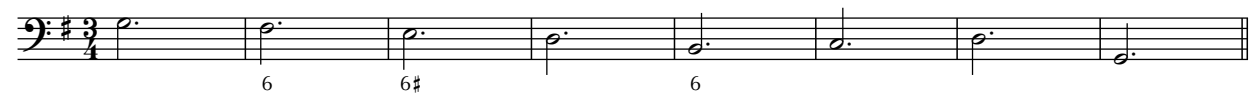

Beispiel 5: Georg Friedrich Händel, Chaconne HWV 435 und Chaconne aus der Suite G-Dur HWV 442; Johann Sebastian Bach, Goldberg-Variationen

15 Caplin 2004. 


\section{Toccata, vierstimmig, nach dem Vorbild von Jan Pieterszoon Sweelinck}

Die Wahl der Toccata mag fragwürdig erscheinen, da sie - etwa bei Komponisten wie Girolamo Frescobaldi oder Michelangelo Rossi - ein Ort satztechnischer Experimente zu sein scheint. In der Tat ist es wohl kaum möglich, etwa von den Toccaten Frescobaldis Prinzipien für satztechnische Stilübungen herleiten zu wollen. Ganz anders verhält es sich aber mit den Toccaten Sweelincks, die sowohl in formaler Hinsicht als auch, was ihre satztechnischen und harmonischen Mittel anbelangt, sich hervorragend dazu eignen.

- Form, nach dem Muster antiker Rhetorik: Exordium, Narratio, Argumentatio, Conclu$\mathrm{sio}^{16}$. Harmonische Frequenz und Dramaturgie

Auch wenn eine genaue wissenschaftliche Begründung hier nicht erfolgen kann ${ }^{17}$ : Der aus der Antike herrührende klassische Aufbau der Rede scheint vielen, wenn nicht gar den meisten Toccaten Sweelincks zugrunde zu liegen. Der prinzipielle formale Aufbau von Einleitung (Exordium), zwei unterschiedlichen Kernteilen (Narratio und Argumentatio) und Schlussteil (Conclusio) ist ein hilfreiches Formmodell für die Analyse und für die Stilkopie. Neben dieser teiligen Anlage ist in den Sweelinckschen Toccaten insofern eine übergreifende Dramaturgie zu erkennen, als die rhythmische Dichte kontinuierlich zunimmt, während die Frequenz der Klangfortschreitungen, vor allem gegen Ende der Argumentatio, deutlich abnimmt.

- Formale Funktion von Harmonik

Die Differenzierung unterschiedlicher Arten von Harmonik, die mit dem Frühbarock einsetzt, zeigt sich in der Sweelinckschen Toccata sehr deutlich: Im Exordium dominiert Prolongationsharmonik, oft durchsetzt mit Sequenzen, die beiden Kernteile sind beinahe ausschließlich durch Sequenzharmonik geprägt und die Conclusio besteht meist aus einer Kadenz und einer sich daran anschließenden stark figurierten plagalen Wendung. Alle Formteile werden mittels Kadenzharmonik beendet.

- Sequenzmodelle und deren Figuration

Es findet sich eine große Vielfalt an Sequenzmodellen. Traktate wie die von Spiridionis (1670/71)) stellen den (nachträglichen) Versuch dar, diese bereits um 1600 nachweisbaren Sequenzen zu systematisieren, was immer vom Bass ausgehend geschieht. Bei Sweelinck sind diejenigen Modelle vorherrschend, die mit Quintbeziehungen arbeiten: Quintfall oder -anstieg oder eine anderweitige Sequenzierung von Quintoder Quartfällen. Viele Passagen beruhen ausschließlich darauf, dass Sweelinck fünf Quinten nach unten und dann wieder nach oben geht. Dabei ist zu beachten, dass beim Quintfall, resp. Quartanstieg, leittönig wirkende große Terzen verwendet werden, während beim Quintanstieg, resp. Quartabstieg, die natürlichen oder sogar Mollterzen verwendet werden. ${ }^{18}$

16 Ueding 1995, $72 \mathrm{ff}$.

17 Ich beschränke mich darauf, auf den Beitrag von Bettina Varwig in dieser Ausgabe hinzuweisen.

18 Darauf weist auch Francesco Bianciardi ausdrücklich hin (1607, 7). 
- Echo-Technik

Eine typisch frühbarocke Technik stellt der Einsatz von Echos dar. Entscheidend erscheint mir hier zum einen die Erkenntnis, dass das Echo - satztechnisch gesehen - eine besondere Form der Imitation darstellt und dass mehrere Möglichkeiten unterschieden werden müssen: Echo im Oktavabstand, rein dynamisches Echo (= satztechnische Wiederholung), Echo mit eingebauter Imitation sowie die unterschiedlichen Verhältnisse zwischen Echo-Motiv und harmonischer Fortschreitung.

- Vergleichender Blick: Frescobaldi

Nach einer intensiven Beschäftigung mit Sweelinckschen Toccaten darf nicht in Vergessenheit geraten, dass eine etwas später entstandene Toccata bei Frescobaldi - sicherlich dem zentralen Meister der Toccata für Tasteninstrumente - deutlich anders aussehen kann. Dass das Attribut ıbarockı zu Recht auch auf Musik angewendet werden kann, zeigt Frescobaldis Vorliebe für unregelmäßige Formung ebenso wie der ausgiebige Gebrauch von verminderten und übermäßigen Intervallen und noch für uns heute atemberaubenden Dissonanzbildungen. Einmal mutmaßten die Studenten, dass die Begegnung mit Frescobaldi für Besucher aus dem `Norden` ein gehöriger Kulturschock gewesen sein musste. Die Bedeutung von Komponisten wie Frescobaldi zu erkennen und Zugang zu ihrer Musik zu finden - auch dies wäre ein lohnendes Ziel einer satztechnischen Übung.

\section{Schlussbemerkung}

Diese kleine Präsentation eines Lehrgangs ist zu verstehen als Zwischenbericht einer sich ständig wandelnden Konzeption. Angesichts der Tatsache, dass die Improvisation sowohl in der Didaktik als auch der Praxis des 17. Jahrhunderts eine kaum zu unterschätzende Rolle gespielt hat, sollte auch dieser Bereich integriert werden. Dazu eignen sich vor allem Improvisationen über Ostinato-Bässe, bei fortgeschrittenen Studenten kämen auch Toccata-Improvisationen über gegebene Bässe in Frage. Improvisation und die mit ihr zusammenhängende Didaktik prägen einen ganz eigenen Bereich aus; deshalb wurde dieser Aspekt hier (noch) weitgehend ausgeklammert.

Gerade im 17. Jahrhundert zeigt sich, dass Theorie der oft schwierige, aber doch unverzichtbare Versuch ist, Ordnung ins Chaos zu bringen, um einerseits Erkenntnisse über die Musik zu gewinnen und um andererseits gelungene Praxis, sei es als Aufführung oder in der Stilkopie, möglich zu machen. Vielleicht ist das 17. Jahrhundert auch deshalb so interessant, weil es angesichts der beträchtlichen Neuerungen und Umbrüche, die sich in ihm zutrugen, ähnliche Probleme der Theoriebildung aufwarf und immer noch -wirft wie die Musik nach 1900: mit dem Unterschied vielleicht, dass man damals um eine praxisorientierte Theoriebildung zuweilen mehr bemüht war als heute. Auch in diesem Sinne kann die Beschäftigung mit der Satztechnik des 17. Jahrhunderts nur von Nutzen sein. 


\section{Literatur}

Bernhard, Christoph (2003), Die Kompositionslehre Heinrich Schützens in der Fassung seines Schülers Christoph Bernhard, hg. von Joseph Müller-Blattau, Kassel u.a.: Bärenreiter.

Bianciardi, Francesco (1607), Breve Regola per imparar'a sonare. http://www.bassus. cmusge.ch/bianciardi/bianciardi.html

Caplin, William E. (2004), „Zur Klassifizierung harmonischer Fortschreitungen«, in: Musiktheorie zwischen Historie und Systematik, hg. von Ludwig Holtmeier, Michael Polth und Felix Diergarten, Augsburg: Wißner, 245-253.

Dirksen, Peter (1997), The Keyboard Music of Jan Pieterszoon Sweelinck, Utrecht.

Gasparini, Francesco (1708), L'armonico pratico al cimbalo, Reprint Bologna: Forni 2005.

Hammond, Frederick (1983), Girolamo Frescobaldi. His Life and Music, Cambridge, MA: Harvard University Press.

Hirschmann, Wolfgang (2005), „Das 17. Jahrhundert: Desintegration und Diversifizierung", in: Musiktheorie (= Handbuch der Systematischen Musikwissenschaft 1), hg. von Helga de la Motte-Haber und Oliver Schwab-Felisch, Laaber: Laaber.

Menke, Johannes (2004), »17. Jahrhundert im Kontrapunktunterricht - ein Plädoyer«, Musiktheorie 19, 385-390.

Muffat, Georg (1699), Regulae concentuum Partiturae. http://www.bassus.cmusge.ch/ muffat/muffat-regulae.html

Nottebohm, Gustav (1873), Beethoven's Studien, Leipzig: Breitkopf \& Härtel.

Owen, Harold (1992), Modal and Tonal Counterpoint, New York: Schirmer.

Schönberg, Arnold (1946), „Kriterien für die Bewertung von Musik«, in: Arnold Schönberg, Stil und Gedanke, Frankfurt a. M.: Fischer 1992, 171-184.

Spiridionis a Monte Carmelo (1670/71), Nova instructio pro pulsandis organis spinettis, manuchordis etc., Bamberg, Reprint Colledara: Andromeda 2003.

Ueding, Gert (1995), Klassische Rhetorik, München: Beck. 